\title{
Phase Averaging Analysis of the Unsteady Velocity Profiles in the Separation Flow on the Flat Plate"
}

\author{
Masaki YAMAGISHI $^{* *}$, Tomoko TOGANO ${ }^{* * *}$ and Shinichi TASHIRO ${ }^{* * * *}$ \\ **Nagaoka National College of Technology, \\ 888 Nishikatakai, Nagaoka, Niigata, 940-8532 JAPAN \\ E-mail: yamagisi@nagaoka-ct.ac.jp \\ ${ }^{* * *}$ Otsuma Women's University, \\ 2-7-1 Karakita, Tama, Tokyo, 206-8540 JAPAN \\ ****Tokyo Metropolitan University, \\ 1-1 Minamiosawa, Hachioji, Tokyo, 192-0397 JAPAN
}

\begin{abstract}
Turbulent separated flow structure was studied by the phase averaging analysis to avoid the difficulty in the data acquisition of the phase angle of the time dependent signal with frequency modulation. This study proposed a new method of phase averaging by the wavelet transformation, and compared the results of the phase averaging by the wavelet transformation with those by the traditional phase averaging technique. These two techniques of the phase averaging, using the experimental data of the velocities in a periodically fluctuating separated flow, showed noticeable difference between their results. If the velocity signals include the frequency modulation, many shapes of velocity profiles by the traditional phase averaging technique will be nearly the same, and be similar to those of the time-averaged velocity. The wavelet transformation, however, showed the clear difference in the velocity profiles at each phase angle even for the velocity signals with modulated frequency.
\end{abstract}

Key words: Phase Averaging, Wavelet Transformation, Periodic Fluctuation, Separated Flow, Shear Layer

\section{Introduction}

Separation phenomenon in a flow becomes the source of oscillation and noise which lead to the crucial damage of the performance of machines. The control and the reduction of separated flow are, therefore, the most significant subjects in the engineering. From the point of view of the controlling and reducing the flow separation, introducing an external fluctuation or forcing is the promising method because the periodic external fluctuation causes dynamic changes of the flow pattern in the downstream separated region. Recently, by introducing the external fluctuation or forcing, many studies have been conducted aiming to the reduction of the separated flow region. In the weak external fluctuation, the disturbance of the frequency develops in the separated shear layer, and has high growth rate due to the Kelvin-Helmholts instability ${ }^{(1)(2)}$. The rolling up of the shear layer generates discrete vortices with instability frequency. The vortices due to the rolling up of the separated shear layer will promote the flow reattachment. On the other hand, in the fluctuation with large amplitude, the fluctuation will cause the fluttering of the separated shear layer, and also the rolling up of the separated shear layer with the frequency of the

*Received 23 July, 2007 (No. T1-04-0385) Japanese Original : Trans. Jpn. Soc. Mech. Eng., Vol.71, No.702, B (2005), pp.450-458 (Received 6 Apr., 2004) [DOI: 10.1299/jee.2.667] 
external fluctuation. Sigurdson and Kiya et al. suggested that the effective frequency on the reduction of the separated region is multiples of the frequency of the shedding vortex from the reattachment point ${ }^{(3)(4)}$. In the frequencies by which the separated region is reduced, the initial separated shear layer will fluctuate dynamically, and thereby forming the vortices periodically ${ }^{(5)}$.

We have investigated experimentally and numerically the effect of the periodic external fluctuation of the flow velocity on the reduction of the separated region above the inclined flat plate ${ }^{(6)(7)}$. The present study aims to clarify the fluttering motion of the separated shear layer due to the introduced external fluctuation of the flow velocity. Although our numerical simulation revealed the fluttering motion of the separated shear layer, to confirm it in the actual flow, the phase averaging analysis was applied to the data of velocity signals in the periodically fluctuated separated flow region in the present study. Furthermore, the method of the wavelet transformation was applied to the phase averaging of the velocity signals with frequency modulation ${ }^{(8)}$. The result of phase averaging by the wavelet transformation was compared with that by the traditional phase averaging technique, thereby discussing also the change of the flow structure in the fluctuated separated region.

\section{Definitions of phase averaging techniques}

In the turbulent flow with an introduced external periodic fluctuation or external periodic forcing as the present experiment, the instantaneous velocity $u$ can be decomposed into the three levels;

$$
u=U+\tilde{u}+u^{\prime} .
$$

Where the components; $U, \tilde{u}, u^{\prime}$ denote the time-averaged, the fluctuation with the same frequency as that of the introduced fluctuation, and the fluctuation with the other frequencies, respectively ${ }^{(9)}$. The fluctuating components; $\tilde{u}$ and $u^{\prime}$ are called as a periodic and a turbulent component in this paper. A coherent structure means the structure by the phase-averaged components, while an incoherent structure is due to the random components. Here coherent structure is denoted as follows;

$$
\langle u\rangle=U+\tilde{u} .
$$

In order to clarify the periodic motion of the external forcing flow, we applied the method of phase averaging to the data of velocity of the separated flow with a periodic fluctuation.

The traditional phase averaging technique is simply to average the pre-periodic signals $u(t)$ of the same phase angles. The phase angle is referred to that of the reference signals $u_{R}(t)$, which has same periodicity with the signals $u(t)$ and are measured with them simultaneously ${ }^{(10)}$. The phase angle is obtained by dividing a cycle of the reference signal into regular intervals. A cycle of the signal is determined by the interval between the neighboring maxima of the signal (10)(11). If the signals modulated in frequency or amplitude, the determination of the interval of the neighboring intersection of the reference signal and its time averaged value to a half cycle is a good application ${ }^{(12)}$. The interval of the time in which the amplitude of the reference signal becomes a half of maxima is also used for the determination of a cycle ${ }^{(13)}$. In the present study, as the traditional technique, a half cycle of the reference signal is determined by the interval between the neighboring maximum and minimum of the reference signal. Figure 1 shows the process of the determination of the phase angle by the traditional technique. The phase angle $\theta$ at the time $t$ is defined by the equation as follows ${ }^{(12)(14)}$; 


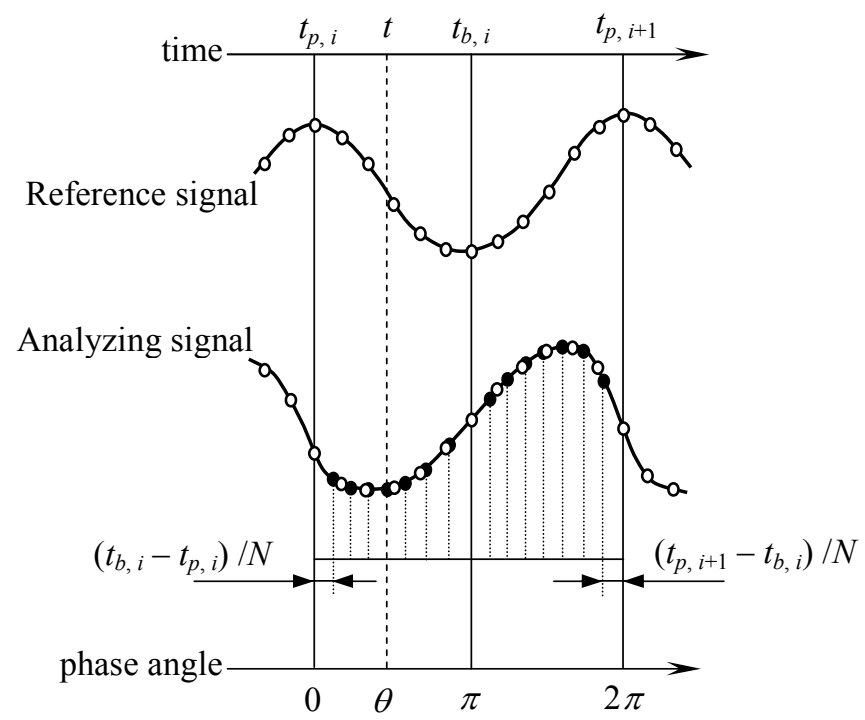

Fig.1 Traditional phase averaging ( $\circ$ : real data, $\bullet:$ interpolated data )

$$
\begin{array}{ll}
\theta=\pi \frac{t-t_{p, i}}{t_{b, i}-t_{p, i}} & \left(t_{p, i} \leq t \leq t_{b, i}\right) \\
\theta=\pi \frac{t-t_{b, i}}{t_{p, i+1}-t_{b, i}}+\pi & \left(t_{b, i} \leq t \leq t_{p, i+1}\right)
\end{array}
$$

Here $t_{p, i}$ is the time in which the reference signal $u_{R}$ satisfies the conditions $u_{R}>0$ and $d u_{R} / d t=0 . \quad t_{b, i}$ is the time in which the reference signal $u_{R}$ satisfies the conditions $u_{R}<0$ and $d u_{R} / d t=0$. The suffix $i$ is the number of cycle from $t=0$. The interval $t_{b, i}-t_{p, i}$ is the half cycle, and $t_{p, i+1}-t_{b, i}$ is the next half cycle. From the Eqs. (3) and (4), the time of the signals $u(t)$ at the phase angle $\theta$ is defined as follows;

$$
\begin{array}{ll}
t=t_{p, i}+\frac{t_{b, i}-t_{p, i}}{\pi} \theta & (0 \leq \theta \leq \pi) \\
t=t_{b, i}+\frac{t_{p, i+1}-t_{b, i}}{\pi}(\theta-\pi) & (\pi \leq \theta \leq 2 \pi)
\end{array}
$$

In the case of determination of the phase angle by dividing a half cycle into regular intervals, these equations are rewritten as

$$
\begin{array}{ll}
t=t_{p, i}+\frac{t_{b, i}-t_{p, i}}{N} n & (0 \leq \theta \leq \pi) \\
t=t_{b, i}+\frac{t_{p, i+1}-t_{b, i}}{N} n, \quad(\pi \leq \theta \leq 2 \pi)
\end{array}
$$

where $n=0,1,2, \ldots, N-1$. The number $N$ is 8 in the present study. Namely, a cycle is 
divided into 16 intervals. The instantaneous velocities at the particular phase angle determined by the method described above are obtained by the interpolation. Then the velocity values which are obtained at each cycle are averaged.

In the traditional phase averaging technique described above, phase differences will be appeared if the velocity signals include the frequency modulation. Also the differences of the measurement position between the points obtained the analyzing velocity signal and the reference signal will make the problem of 'jittering'. These phase jitters often make 'washout' of the phase-averaged data ${ }^{(15)}$. In the present study, therefore, the velocity signal itself was used for the reference signal to avoid the washout. Namely, the velocity signal was averaged at the same phase angle as that of the extracted reference signal. Togano et al. and Kiya et al. obtained the reference signal through the fourier transformation of the velocity signal which is filtered by a narrow band pass filter ${ }^{(14)(16)}$. The phase angle is obtained by dividing a cycle of the reference signal into regular intervals.

We used the wavelet transformation in detection of the modulated frequency. Figure 2 shows the schematic process of the phase averaging. The instantaneous maxima in the wavelet coefficients must be obtained to determine the dominant frequency on each time. The maxima of the wavelet coefficient are detected in that envelope which expressed the absolute coefficient. To obtain the absolute coefficient, a complex-type the wavelet is therefore required. By using Morlet's wavelet ${ }^{(17)}$, which is one of the complex-type wavelet, the dominant frequencies can be detected in the narrow band around the external fluctuation frequency $f_{e}$.

The wavelet coefficients are not proportional to the amplitude of each frequency component. If Morlet's wavelet, shown as bellow, is used;

$$
\psi(t)=e^{i k t} \cdot e^{-\left(t t^{2} / 2\right)}, \quad k=6
$$
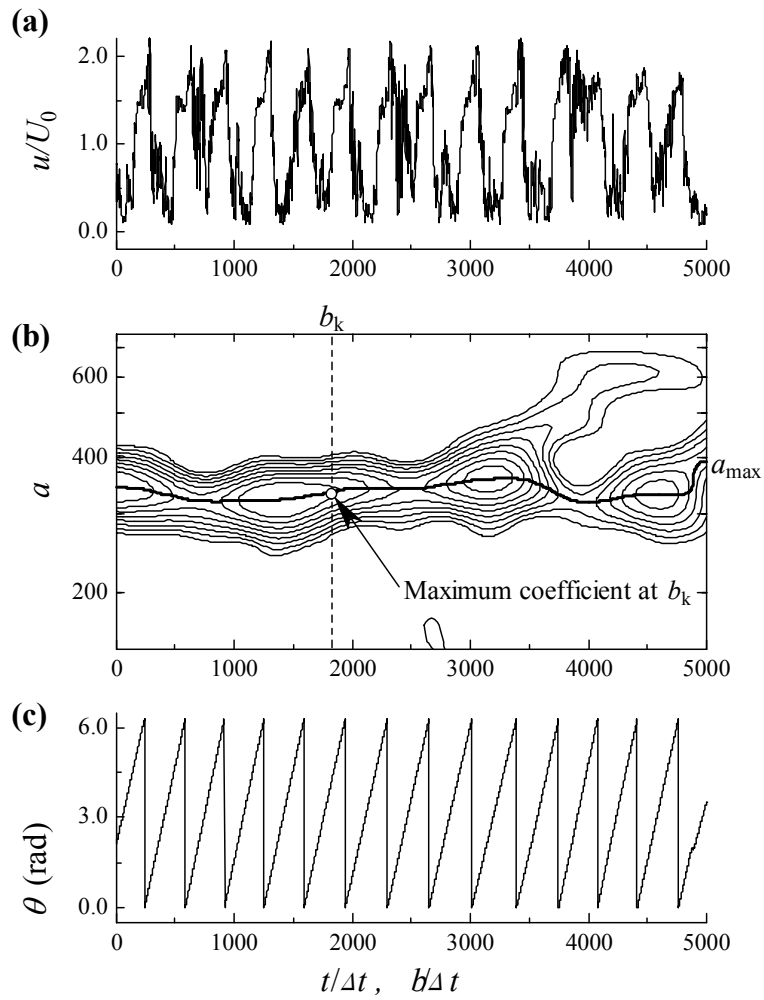

Fig.2 Phase averaging by using wavelet transformation 
the coefficients divided by $\sqrt{a}$ are proportional to the amplitude. In the present study, the wavelet transformation equation for the fluctuation velocity $u(t)$ is therefore defined as follows;

$$
w f(b, a)=\frac{1}{\sqrt{a}} \int_{-\infty}^{\infty} u(t) \frac{1}{\sqrt{a}} \overline{\psi\left(\frac{t-b}{a}\right)} d t .
$$

If $\psi(t)$ satisfies the admissibility condition, the reconstruction formula of the wavelet transformation can be written as follows;

$$
u(t)=\frac{1}{C_{\psi}} \int_{0}^{\infty} \int_{-\infty}^{\infty} w f(b, a) \psi\left(\frac{t-b}{a}\right) \frac{d a d b}{a^{2}} .
$$

If Morlet's wavelet is used, Equation (11) is rewritten as

$$
u(t)=\frac{1}{C_{\psi}} \int_{0}^{\infty} \int_{-\infty}^{\infty}(W \cos \alpha+i W \sin \alpha) \cdot \psi\left(\frac{t-b}{a}\right) \frac{d a d b}{a^{2}} .
$$

Where $W$ and $\alpha$ are shown as follows;

$$
\begin{aligned}
& \begin{array}{l}
W=|w f(b, a)|=\sqrt{w f_{R}(b, a)^{2}+w f_{I}(b, a)^{2}} \\
\alpha=\tan ^{-1} \frac{w f_{I}(b, a)}{w f_{R}(b, a)} \\
\quad\left(w f_{R}(b, a)=\operatorname{Re}[w f(b, a)], \quad w f_{I}(b, a)=\operatorname{Im}[w f(b, a)]\right)
\end{array}
\end{aligned}
$$

Actually the inverse transformation as shown Eq. (12) is impossible because Morlet's the wavelet is not orthonormal. However it is remarkable that the real part and imaginary part of the wavelet coefficient, $w f_{R}$ and $w f_{I}$, are rewritten in the forms respectively as shown bellows;

$$
\begin{aligned}
& w f_{R}(b, a)=W \cos \alpha \\
& w f_{I}(b, a)=W \sin \alpha .
\end{aligned}
$$

For the reference signal, we used the real part of the wavelet coefficients of the dominant frequency component on each time. Figure 2(b) shows the contour line of the absolute the wavelet coefficients. The absolute wavelet coefficients become the maximum at the dominant frequency $a_{\max }$. The reference signal, shown in Fig. 2(b) by the bold solid line, is extracted by the detection of the instantaneous maximum $W_{\max }$ at the frequency $a_{\max }$ on each time. The instantaneous maximum $W_{\max }$ is the largest value in the detection band and the value satisfying the condition of $d^{2} W_{\max } / d a^{2}<0$. If there is not the wavelet coefficient which satisfies those conditions in detection, the data of velocity at that time is rejected in the process of the phase averaging. After the detection, the reference signal $u_{R}(t)$ can be shown as Eq. (17).

$$
u_{R}(t)=W f_{R}\left(b, a_{\max }\right)=W_{\max } \cos \{\theta(b)\}
$$


Here the phase angle $\theta(b)$ is shown as Eq. (18).

$$
\theta(b)=\tan ^{-1} \frac{w f_{I}\left(b, a_{\max }\right)}{w f_{R}\left(b, a_{\max }\right)}
$$

The phase angle $\theta(b)$ is shown in Fig. 2(c) obtained by the real part $w f_{R}\left(b, a_{\max }\right)$ and the imaginary part $w f_{I}\left(b, a_{\max }\right)$ of the wavelet coefficients. It is the advantage of this method that the phase angles of the reference signal on each time can be directly obtained. Phase averaging is conducted by averaging the signals $u(t)$ at the same phase angle $\theta(b)$.

As the velocity signals were not measured simultaneously at each measurement point in the separated flow region, the different phase angles were obtained at each measurement point. To estimate the phase-differences between the measurement points, another periodic velocity was measured at the fixed point. This periodic velocity and the velocity in the separated flow region were measured simultaneously. The phase-differences are estimated by the cross-correlation between these two velocity signals ${ }^{(18)}$. The phase-differences on each point are added to the phase angles $\theta(b)$ in the phase averaging process through the wavelet transformation. The periodic velocity signals measured at the fixed point were also used for the reference signals at the traditional phase averaging technique.

\section{Experimental apparatus}

The experiments were performed in the wind tunnel of open-jet type; $200 \mathrm{~mm} \times 200$ $\mathrm{mm}$ in cross section ${ }^{(6)}$. Figure 3 shows the schematic illustration of the test section. The separated flow is formed on the inclined flat plate. The flat plate is acrylic plate $150 \mathrm{~mm}$ lengths and $10 \mathrm{~mm}$ thick, of which the leading edge is sharpened like a knife-edge. The flat plate is set with 5 degrees to the mean flow. The external periodic fluctuation which is introduced in the separated flow region is generated in the wake of the bluff body; the "generator" set in the upstream flow. The generator can be set with various projectional sections perpendicular to the flow. The frequency of the fluctuation generated by the generator is in the range of $10 \sim 140 \mathrm{~Hz}$ at the free-stream velocity $U_{0}=3 \mathrm{~m} / \mathrm{s}$.

The free-stream velocity; $U_{0}$ was fixed at $3 \mathrm{~m} / \mathrm{s}$ through the experiment. Reynolds number is 30,000 based on the free-stream velocity; $U_{0}$ and on the length of flat plate; $L$. In our previous experiments, the frequencies of the external fluctuation were set at the multiples of the characteristic frequencies in the separated flow region ${ }^{(6)(7)}$. It was found that $14 \mathrm{~Hz}$ was the predominant frequency measured in the separated flow region without

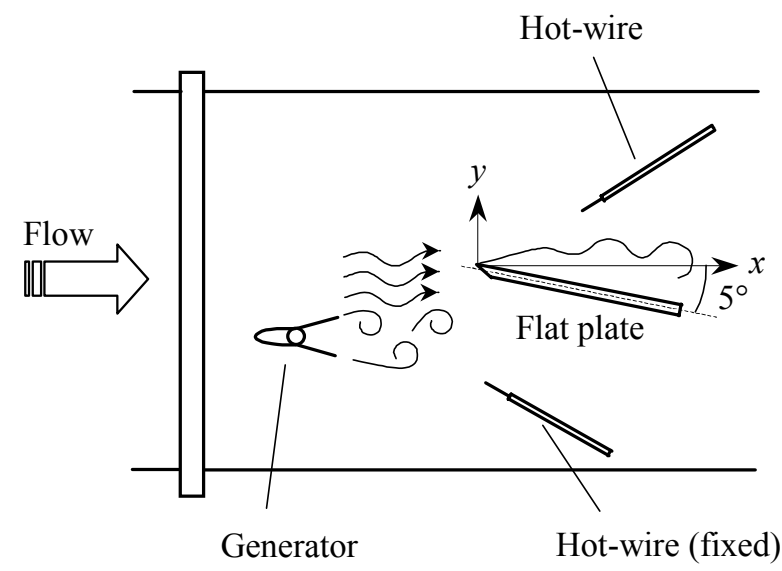

Fig.3 Experiment apparatus 
fluctuation and was the most effective to reduce the separation of flow. In the separated shear layer without fluctuation, the remarkable peak of the spectrum was measured at the frequency $f=36 \mathrm{~Hz}$. Furthermore it is also found that the frequency which is greater than about $50 \mathrm{~Hz}$ was less effective on the reduction of the separated region. In the present study, therefore, we chose three frequencies: $f_{e}=14,36$ and $72 \mathrm{~Hz}$ for the external fluctuation frequency. These three frequencies correspond to the Strouhal number; $S t=$ $f_{e} L / U_{0}=0.7,1.8$ and 3.6, respectively, where $U_{0}$ is the free-stream velocity and $L$ is the length of flat plate.

A hot-wire anemometry of constant-temperature type with a single wire probe was used to measure the fluctuation velocity in the streamwise direction. The output signals of the hot-wire anemometer were stored in a digital oscilloscope through A/D converter. The $x$ and $y$-axes in the coordinate system correspond to the streamwise and the normal to the direction of mean flow respectively. The origin of axes is set at the leading edge of the flat plate. Flow velocities were measured at the region in the range from $x=0$ to $30 \mathrm{~mm}$ with an interval of $5 \mathrm{~mm}$. In the wake of the generator, the external fluctuation is generated with the coherent structure and introduced into the separated flow region. To conduct the conditional sampling for the phase averaging, another periodic signal is required for simultaneous measurement. For measuring the periodic fluctuation velocities in the wake of the generator, another hot-wire probe was also set under the flat plate accordingly. This probe was fixed at the point where $x=0 \mathrm{~mm}$ for the sampling of the velocity signals with clear periodicity. Through the band-pass filter, the components of high frequency and DC in these periodic velocity signals were removed. Conditional sampling was triggered by zero-cross condition of these filtered signals. These signals were used also in the analysis to conduct the traditional phase averaging and to obtain the spatial phase-differences due to the different measurement points.

\section{Results and discussion}

\subsection{The changes of the velocity profiles by introduction of the fluctuation}

Figure 4 shows the profiles of time-averaged velocity in the separated region without and with fluctuation along $y$-axis at $x / L=0.1$. In the reversed flow region near the wall, no correction was made in the reading of hot-wire. The inflection point of the velocity profile, shown here, indicates the separation of flow with no fluctuation. By introducing the external fluctuation into the separated flow region, averaged flow velocity near the wall increases. The velocity profile at the frequency of $S t=0.7$ has convex shape, which indicates the disappearance of the separated flow region. The velocity profiles at the frequencies of $S t$ $=1.8$ and 3.6 still have inflection point. Namely these frequencies are less effective on the reduction of the separated flow region than the frequency of $S t=0.7$.

\subsection{Comparisons of the techniques of the phase averaging}

Figure 5 shows the phase-averaged velocity waveforms in a unit cycle by using the traditional technique and those by using the wavelet transformation at the frequency of $S t=$

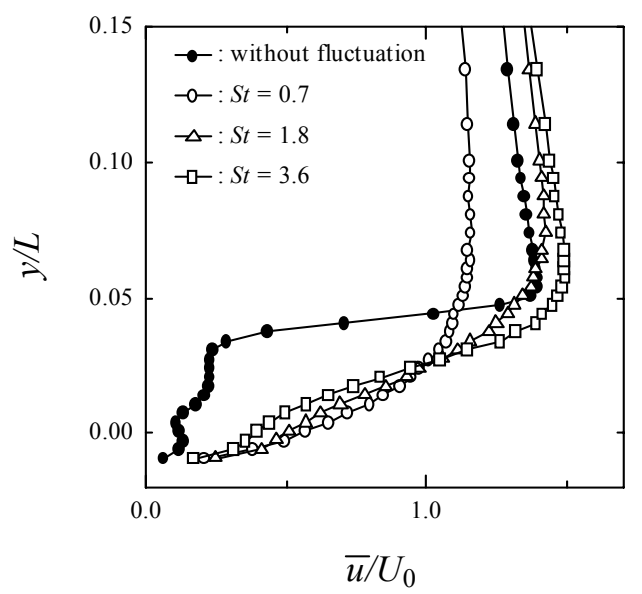

Fig.4 Time-averaged velocity profiles at $x / L=0.1$ 
0.7 and 3.6. The bold black line in the figure indicates the time-averaged velocity. The results at the frequencies of $S t=1.8$ were nearly the same at the frequencies of $S t=3.6$. In these results, the phase angle at which each reference signal become maximum is set at $\theta=0$. At the frequency of $S t=0.7$, as shown in Fig. 5 (a), the phase-averaged velocity by both techniques show almost same results. At the frequency of $S t=3.6$, as shown in Fig. 5(b), the amplitude of phase-averaged velocity by using the traditional technique was smaller than that by using the wavelet transformation technique. The values of the phase-averaged velocity at all phase angles therefore resembles each other and similar to those of time-averaged velocity. In the use of the traditional technique, the washout occurred. On the other hand, by using the wavelet transformation, the phase-averaged velocities show large amplitude and show clear periodicity. From these results, it is found that the phase averaging by using the wavelet transformation could avoid the washout of the averaged data.

Figures 6 and 7 show the profiles of phase-averaged velocities along $y$-axis at $x / L=0.1$. The data of the velocity profiles were processed in the smoothing treatment ${ }^{(19)}$ for only once. In these figures, phase angle $\theta$ is set as zero based on the phase angle of the signal measured at the fixed point. The profiles of time-averaged velocity are also shown by the solid circles. In these figures, (a) and (b) show the results by the traditional technique and by the wavelet transformation respectively. At the frequency of $S t=0.7$, the profiles of phase-averaged velocity in both phase averaging technique are similar to each other, and vary dynamically at each phase. As shown in Fig. 6 , the profile of the phase-averaged velocity varies from convex to concave profiles periodically. In the phase angle $\theta=\pi$, the phase-averaged velocity near the wall is shown to be accelerated extremely. It indicates that the separated shear layer flutters and reattaches at this phase angle. In the velocity profiles by the traditional technique as shown in Fig. 6 (a), there is the point at which the phase-averaged velocity does not change at each phase angle at the point $y / L=0.04$. Around that point, the intermittent fluctuation appears in the velocity signals, and it also causes washout for the results of using the traditional technique. On the other hand, velocity profiles shown by the wavelet transformation have large amplitude around that point. It is found that the phase averaging technique using the wavelet transformation could remove the intermittent fluctuation in the process of the phase averaging. At the frequency of $S t=3.6$, as shown in Fig. 7, the profiles of the phase-averaged velocity are different from those by other technique. The profiles of the phase-averaged velocity by the traditional technique resemble each other and similar to those of the time-averaged velocity. The velocity signals acquired in the separated flow region included frequency modulation, which leads to the washout. The fluttering motion of shear layer can not be seen from those profiles. The velocity profiles 
by the wavelet transformation, however, show clearly the change at each phase angle even if the velocity signals modulated in frequency. The periodic change in the phase-averaged velocity is shown even near the wall.

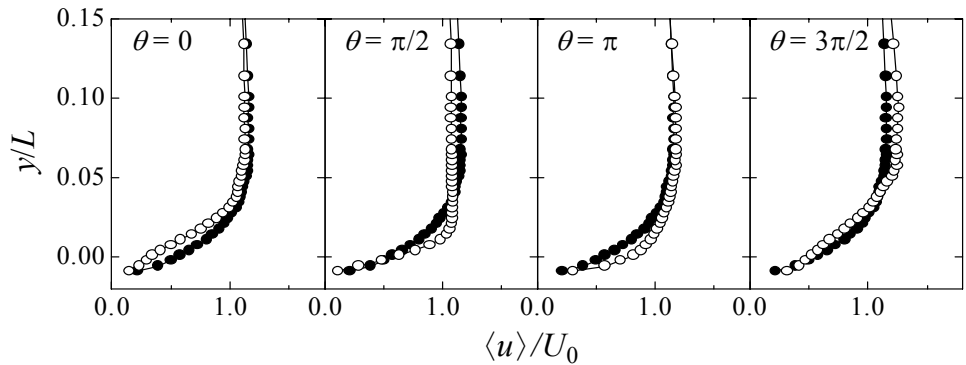

(a) Traditional technique

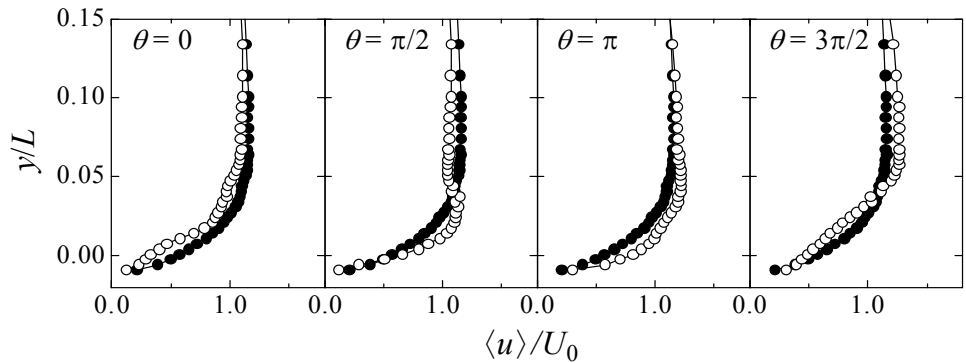

(b) Technique by using wavelet transformation

Fig.6 Profiles of phase-averaged velocity at $S t=0.7$

( $\bullet$ : Time-averaged velocity, $\circ:$ Phase-averaged velocity )

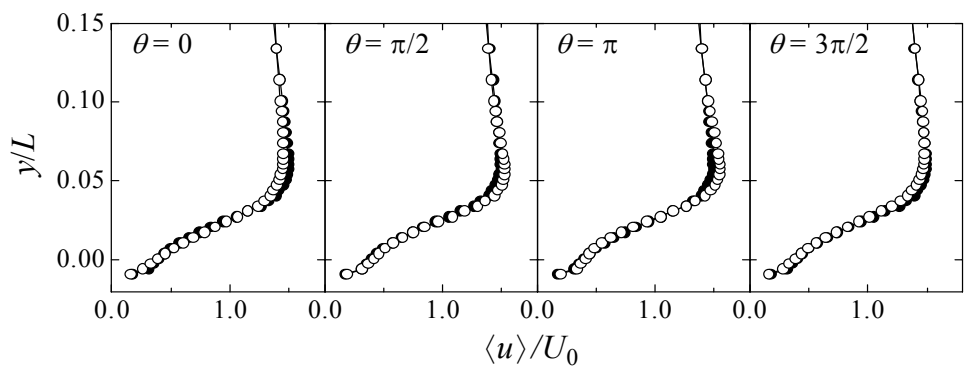

(a) Traditional technique

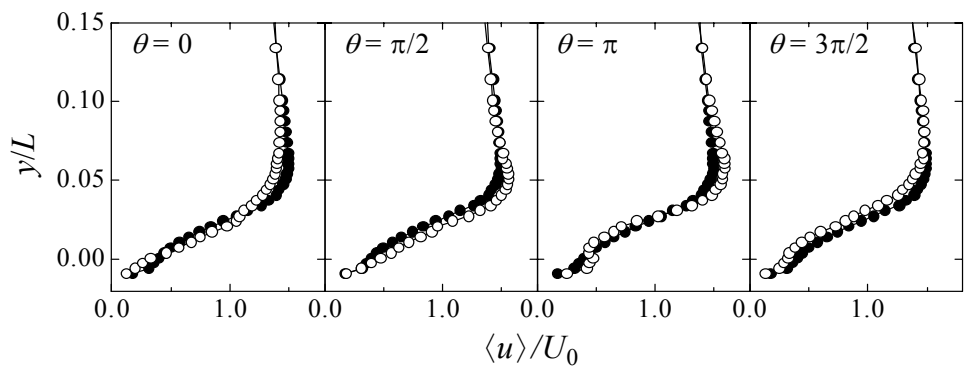

(b) Technique by using wavelet transformation

Fig.7 Profiles of phase-averaged velocity at $S t=3.6$

( $\bullet$ : Time-averaged velocity, $\circ:$ Phase-averaged velocity ) 
Figure 8 shows the profiles of the turbulent intensity in the phase-averaged velocity. The turbulent intensity in the phase averaged velocity is estimated by that of the phase-averaged velocity signals $\langle u\rangle$ given in the phase interval $\Delta \theta=2 \pi / 500$. The solid lines in these figures show the profiles of time-averaged turbulent intensity, where we can see clearly the differences among the other techniques. As shown in Fig. 8(a), by both techniques, the profiles of the turbulent intensity in the phase-averaged velocity have the same shape of curve as that of the time-averaged turbulent intensity at the frequency of $S t=$ 0.7. The positions of which the values become the maxima also correspond to that of the time-averaged turbulent intensity. By the traditional technique, the value of the turbulent intensity decreases at the point $y / L=0.04$. This position; $y / L=0.04$ corresponds to the point shown in Fig. 6(a) where the phase-averaged velocity does not change at each phase angle. The decrease in the turbulent intensity is due to the washout by appearance of the intermittent fluctuation. From the results as shown in Fig. 8(a), however, it is found that the technique using the wavelet transformation could avoid the washout. At the frequency of $S t=3.6$ shown in Fig. 8(b), by the traditional technique, the shape of the profile is very different from that of the time-averaged turbulent intensity. The magnitude of turbulent intensity in the phase-averaged velocity by the traditional technique is smaller than that by using the wavelet transformation in the direction $y$. It is because that the washout occurred at many acquisition points. On the other hand, by using the wavelet transformation, the shape of the profile is similar to that of the time-averaged turbulent intensity. Furthermore the position of which the values become the maximum corresponds to that of time-averaged turbulent intensity.

From the results described above, the phase averaging technique by using the wavelet transformation can avoid the occurrence of the washout of the averaged data. It is concluded that the phase averaging technique by using the wavelet transformation is useful for the investigation of the periodic flow field with frequency modulation.

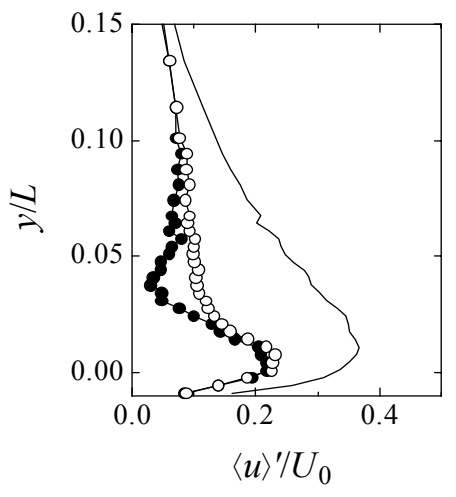

(a) $S t=0.7$

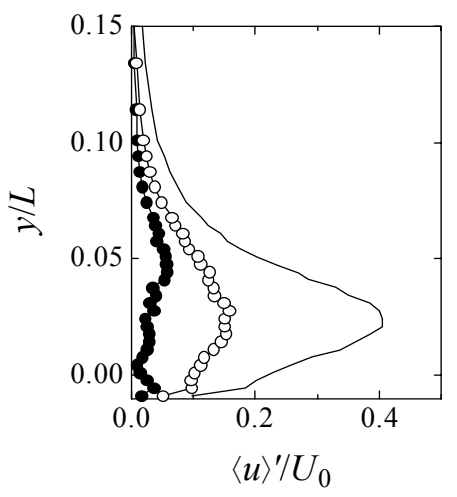

(b) $S t=3.6$

Fig.8 Profiles of phase-averaged turbulent intensity ( $\bullet$ : Traditional technique, $\circ$ : Technique by using wavelet transformation, - : Time-averaged velocity )

\subsection{The periodic motion of the phase-averaged velocity field}

The profiles of the averaged velocity field illustrated by contour lines are shown in Figs. 9 and 10. Figure 9 shows the profiles of the time-averaged velocity and Fig. 10 shows those of the phase-averaged velocity by using the wavelet transformation. From the results of time-averaging as shown in Fig.9, it is found that the averaged velocity near the wall increases. It is indicate the decrease of the separated region. At the frequency of St 
$=0.7$, as shown in Fig. 9(a), the separated region is disappeared. At the frequency of $S t=$ 1.8, the small separated region is seen near the leading edge of the flat plate in Fig. 9(b). The thin separated region is still exist on the wall at the frequency $S t=3.6$ as shown in Fig. 9(c). Furthermore, the velocity outside the shear layer is accelerated when the separated region is exist. From these profiles of time-averaged velocity, however, the periodic motion of the flow field could not be seen.

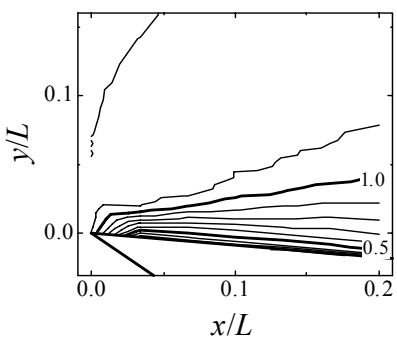

(a) $S t=0.7$

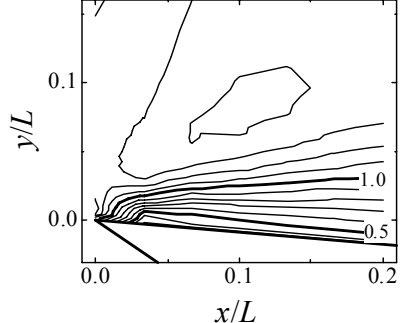

(b) $S t=1.8$

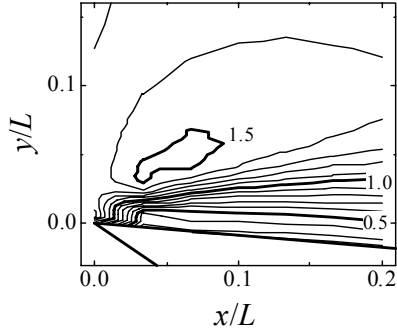

(c) $S t=3.6$

Fig.9 Time-averaged velocity profiles
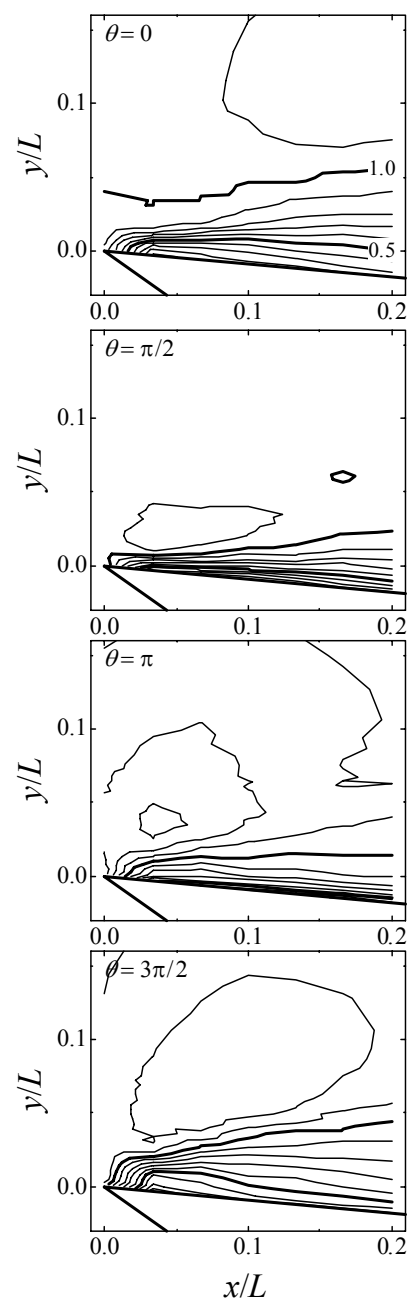

(a) $S t=0.7$
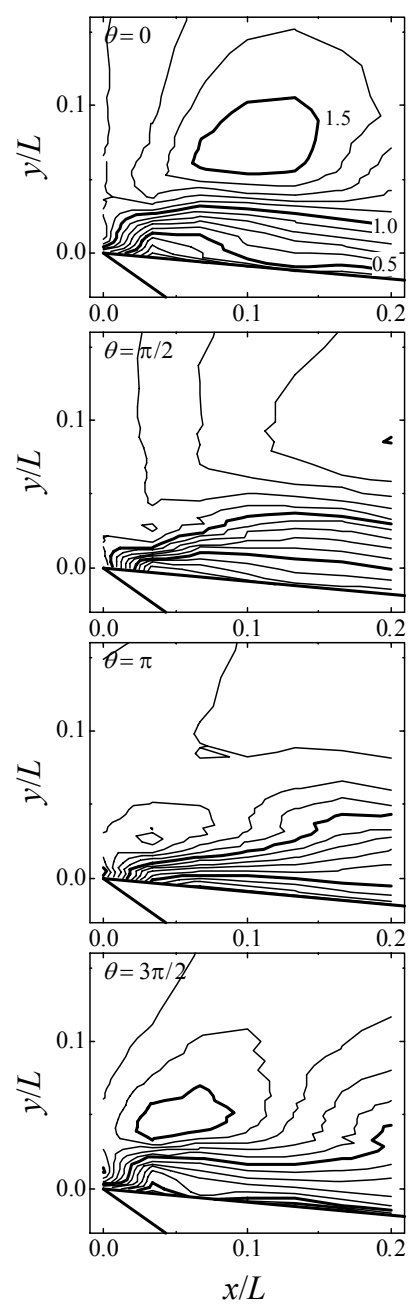

(b) $S t=1.8$
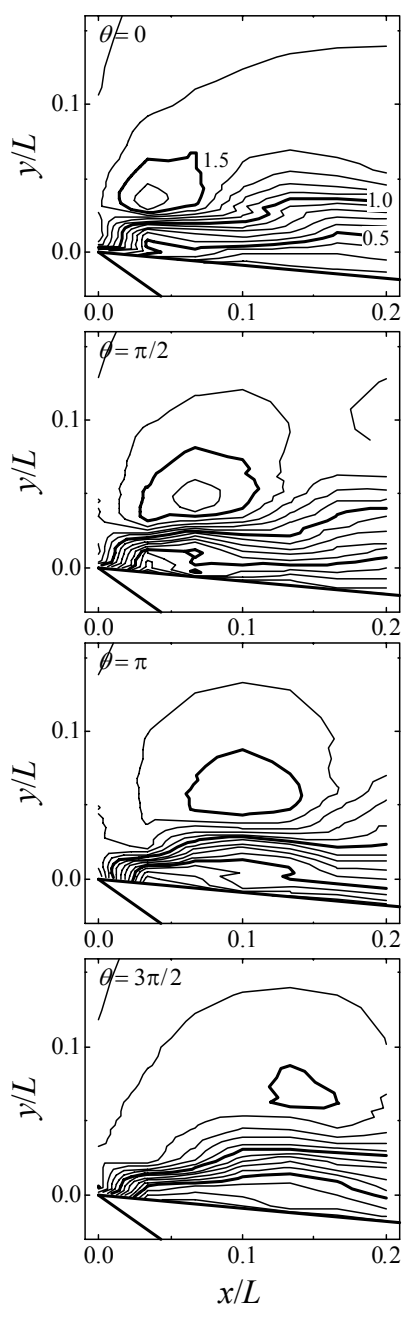

(c) $S t=3.6$

Fig.10 Phase-averaged velocity profiles by using wavelet transformation 
The profiles of phase-averaged velocity are shown in several phase angles during unit period. The layer of the contour lines between $\langle u\rangle=0.5$ and 1.0 indicates the separated shear layer. In Fig. 10, phase angle $\theta$ is set as zero based on the phase angle of the signal measured at the fixed point as like conducting in Figs. 6 and 7. The profiles vary dynamically at each phase and show clearly the periodic motion of the shear layer. At the frequency of $S t=0.7$, as shown in Fig. 10(a), the shear layer flutters dynamically. Furthermore the shear layer is depressed near the wall, and the separated region is disappeared in the phase angle $\theta=\pi, 3 \pi / 2$. In the time-averaged profiles, it is seen that separated region is disappeared. The separated region is, however, existed near the leading edge during the fluttering motion of the shear layer as shown in the phase angle $\theta=0, \pi / 2$. The disappearance of the separated region is shown also at the frequency of $S t=1.8$ in Fig. 10 (b). In the phase angle $\theta=3 \pi / 2,0$, there is a larger separated region than that by the frequency of $S t=0.7$ in the range of $x / L=0 \sim 0.12$. The frequency of $S t=1.8$ has less effective on the reduction of the separated region than that of $S t=0.7$. At the frequency of $S t=3.6$ shown in Fig. 10(c), the separated shear layer shows also fluttering motion. The separated shear layer, however, does not reattach on the wall in the measurement region because the fluttering motion is weaker than that by the other two frequencies. The separated region is seen in all phase angles.

In Fig. 10(a) (c), closed contour patterns are shown outside the shear layer. These patterns are the accelerated velocity region of the external fluctuation. These closed patterns travel downstream along the shear layer. The shear layer is pulled up to the acceleration region of the fluctuation owing to the pressure gradient. The shear layer is to be depressed behind this acceleration region. The aspects of the motion are obviously different from each other with the frequency of fluctuation.

For the comparison, the profiles of phase-averaged velocity by the traditional technique are shown in Fig. 11. At the frequency $S t=0.7,1.8$, at which the acquired velocity signals have strong periodicity and less frequency modulation, the profiles show the same shape which obtained by using the wavelet transformation (Figs. 11(a) and (b)). The differences of the shape of the contour lines are shown around the outer side of the shear layer between Figs. 10 and 11. It is because that washout occurred owing to the appearance of the intermittent fluctuation as described before. At the frequency $S t=3.6$, the profiles are very different from that by using the wavelet transformation. In Fig. 11(c), the periodic motion of the contour lines is very small in the downstream from $x / L=0.1$. Especially, in the separated region between the contour line of $\langle u\rangle / U_{0}=1.0$ and the wall, the profiles are same to that of time-averaged velocity in each phase angle. The results by the traditional technique would lead to the wrong conclusion that the fluttering motion of the shear layer was not caused by the external fluctuation at frequency $S t=3.6$. From comparison of these results, it could be concluded that the phase averaging technique by using the wavelet transformation can obtain the periodic characteristics which was not able to obtain by the traditional technique.

\section{Concluding Remarks}

The phase averaging technique by using the wavelet transformation was proposed and applied to the data of the velocities in the separated flow region. The results by the phase averaging technique were compared with those by the traditional technique. If the velocity signals include the frequency modulation, the amplitude of the phase-averaged velocity by the wavelet transformation technique is larger than that by using the traditional technique. In the process of the phase averaging, the wavelet transformation is useful even for the signals which include frequency modulation. Furthermore, the wavelet transformation can show the dominant frequency among the other frequency components even in the intermittent fluctuation. From the results of the phase averaging by using the wavelet 

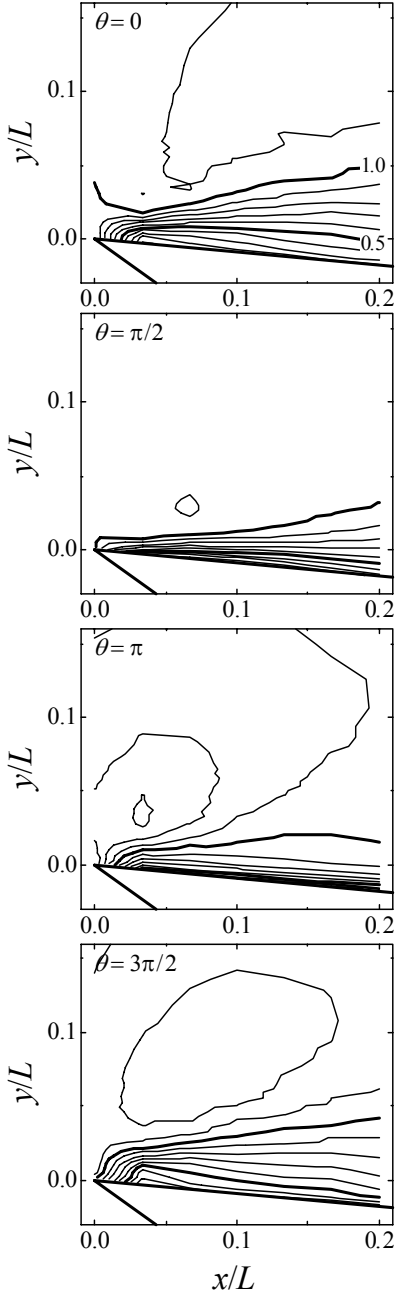

(a) $S t=0.7$
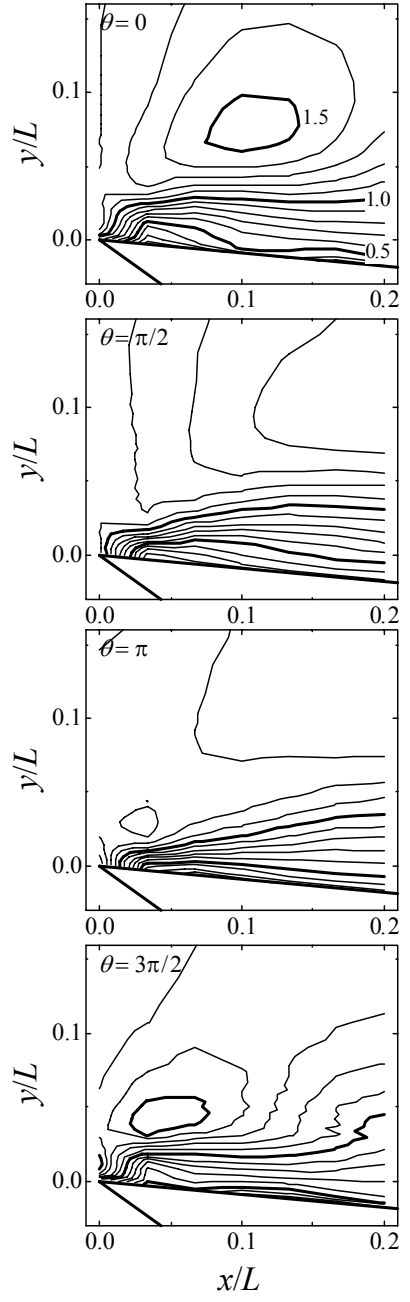

(b) $S t=1.8$
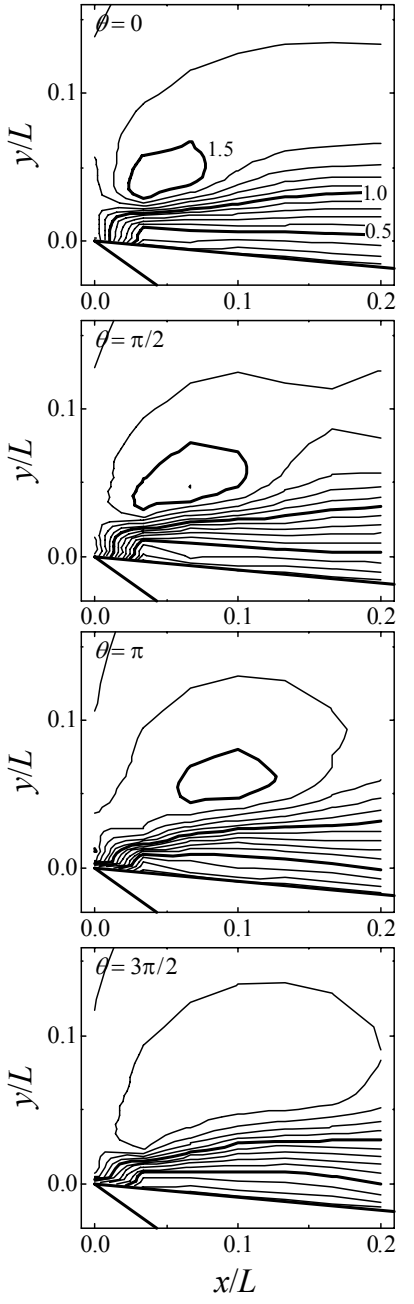

(c) $S t=3.6$

Fig.11 Phase-averaged velocity profiles by traditional technique

transformation, the relationship between the frequency of the external periodic fluctuation and the changes of the organized fluid motion could be discussed. If the suitable frequency of the reduction of the separated region is introduced, the separated flow region will disappear in almost all phases owing to the depression of the shear layer near the flat plate surface. At the other frequencies, however, the depression of the separated shear layer is insufficient because the fluttering is not so strong. It is concluded that the large reduction of the separated flow region is due to the sensitive response of the shear layer to the particular frequency.

\section{References}

(1) Nishioka, M., Asai, M. and Yoshida, S., Control of flow separation by acoustic excitation, AIAA J., vol. 28, No.11, (1990), pp.1909-1915.

(2) Zahman, K. B. M. Q. and McKinzie, D. J., Control of laminar separation over airfoils by acoustic excitation, AIAA J., vol. 29, No.7, (1991), pp.1075-1083.

(3) Sigurdson, L. W., The structure and control of a turbulent reattaching flow, Journal of Fluid Mechanics 298, (1995), pp.139-165. 
(4) Kiya, M., Shimizu, M. and Mochizuki, O., Sinusoidal forcing of a turbulent separation bubble, Journal of Fluid Mechanics 342, (1997), pp.119-139.

(5) Yoshioka, S., Obi, S. and Masuda, S., Organized vortex motion in periodically perturbed turbulent separated flow over a backward-facing step, International Journal of Heat and Fluid Flow, 22, (2001), pp.301-307.

(6) Yamagishi, M. and Tashiro, S., Effect of external fluctuation frequency introduced in the mean-flow on the characteristic frequency of separated flow, JSME International Journal, Series B, Fluids and Thermal Engineering, Vol.45, No.4, (2002), pp.796-803.

(7) Yamagishi, M. and Tashiro, S., Numerical study of the vortex structure in periodically fluctuated turbulent separated flow, Proc. of Conference of Modelling Fluid Flow (CMFF'06), Vol. I, (2006), pp.326-331.

(8) Yamagishi, M., Togano, T. and Tashiro, S., Phase averaging analysis of the unsteady velocity profiles in a separated flow, Proc. of ASME-JSME Joint Fluids Engineering Conference, Fluids Engineering Division Summer Meeting 2003, FEDSM2003-45032 (CD-ROM).

(9) Reynolds, W. C. and Hussain, A. K. M. F., The mechanics of an organized wave in turbulent shear flow. Part 3. Theoretical models and comparisons with experiments, Journal of Fluid Mechanics 54, (1972), pp.263-288.

(10) Davies, M. E., A comparison of the wake structure of a stationary and oscillating bluff body, using a conditional averaging technique, Journal of Fluid Mechanics 75-2, (1976), pp.209-231.

(11) Kiya, M. and Matsumura, M., Incoherent turbulent structure in the near wake of a normal plate, Journal of Fluid Mechanics 190, (1988), pp.343-356.

(12) Kiya, M. and Matsumura, M., Large-scale and small-scale turbulence structure in the intermediate wake of a two-dimensional normal plate (in Japanese), Transaction of Japanese Society of Mechanical Engineering 50, 458, Series B, (1984), pp.2563-2570.

(13) Tanaka, S. and Murata, S., Study on the wake of circular cylinder by using the computational visualization method (in Japanese), Transaction of Japanese Society of Mechanical Engineering 51, 469, Series B, (1985), pp.2831-2845.

(14) Togano, T. and Tashiro, S., A study of Large-scale turbulent field (On procedure of removing of outliers from measurement data and averaging process) (in Japanese), Research Reports of Tokyo Metropolitan Institute of Technology, Vol.11, (1998), pp.221-226.

(15) Perry, A. E. and Wtmuff, J. H., The phase-averaged large structures in three-dimensional turbulent wakes, Journal of Fluid Mechanics 103, (1981), pp.33-51.

(16) Kiya, M. and Matsumura, M., Turbulence structure in the intermediate wake of a circular cylinder (in Japanese), Transaction of Japanese Society of Mechanical Engineering 51, 463, Series B (1985), pp.775-782.

(17) Farge, M., Wavelet transforms and their applications to turbulence, Annual Review of Fluid Mechanics, 24, (1992), pp.395-457.

(18) Obara, H. and Matsudaira, Y., Large vortex formation-mechanism behind wedge under several separation conditions, JSME International Journal, Series B, Fluids and Thermal Engineering, Vol.41, No.4, (1998), pp.788-795.

(19) Taniguchi, S., Deguchi, A. and Miyakoshi, K., Dohda, S., The wake structure of two-dimensional rectangular cylinders having different length-to-width ratios (in Japanese), Transaction of Japanese Society of Mechanical Engineering 54, 498, Series B, (1988), pp.256-264. 